\title{
Predictors of Myocardial Reperfusion in Patients with ST-Elevation Myocardial Infarction Following Thrombolytic Treatment in Rural Community
}

\author{
Patcharaporn UNTAJA ${ }^{1}$, Siriorn SINDHU ${ }^{2, *}$, Patricia Mary DAVIDSON ${ }^{3}$, \\ Orapan THOSINGHA ${ }^{2}$ and Chukiat VIWATWONGKASEM ${ }^{4}$
}

\author{
${ }^{1}$ Royal Thai Army Nursing College, Bangkok 10400, Thailand \\ ${ }^{2}$ Faculty of Nursing, Mahidol University, Bangkok 10700, Thailand \\ ${ }^{3}$ Johns Hopkins School of Nursing, Baltimore 21205, United State of America \\ ${ }^{4}$ Department of Biostatistics, Faculty of Public Health, Mahidol University, Bangkok 10700, Thailand
}

('Corresponding author's e-mail: siriorn.sin@mahidol.edu)

Received: 1 October 2017, Revised: 10 November 2018, Accepted: 30 December 2018

\begin{abstract}
This cross-sectional study aims to examine patient and hospital factors contributing to reperfusion following thrombolysis in patients with ST-segment elevation myocardial infarction (STEMI) in rural hospitals in Thailand. Access to reperfusion therapy for STEMI patients in rural areas is increasing in Thailand, yet clinical outcomes vary between settings and are inferior to recommended guidelines. Ten years after the introduction of thrombolytic treatment for rural patients undergoing STEMI, there is still a limited description of factors influencing the outcomes of thrombolytic treatment.

Data were collected from 87 acute STEMI patients who had received thrombolytic treatment in 12 thrombolysis-capable hospitals located in rural areas. Demographic and clinical characteristics included patient response and treatment seeking behavior, witness response to the patient symptoms, waiting time for treatment, severity of illness, guideline adherence, and thrombolytic reperfusion. The results were: only 31 of 87 patients $(35.63 \%)$ received thrombolytic treatment within $30 \mathrm{~min}$ after arrival at the hospital. A total of 42 patients $(48.28 \%$ ) had successful myocardial reperfusion following thrombolytic treatment. Logistic regression analysis revealed that guideline adherence (OR : $1.58 ; 95 \%$ CI : 1.12 2.25), severity of illness (OR : $0.77 ; 95 \%$ CI : $0.64-0.94$ ), and patient response and treatment seeking behavior (OR : 1.47 ; $95 \%$ CI : 1.01 - 2.04) were predictors of thrombolytic reperfusion. In rural areas, healthcare resources and transfer facilities are limited. Therefore, the improvement of patient awareness is needed. The clinical practice guidelines and consideration of pre-hospital factors should be promoted and the number of thrombolysis-capable hospitals should be increased.
\end{abstract}

Keywords: Thrombolytic reperfusion, ST-elevation myocardial infarction, rural, clinical guidelines, outcomes, access

\section{Introduction}

ST-segment elevation myocardial infarction (STEMI) is a major cause of death and disability for patients with coronary heart disease (CHD) and is part of the clinical spectrum of Acute Coronary Syndrome (ACS). Early restoration of coronary flow by reperfusion therapy, primary percutaneous coronary intervention (PCI), or thrombolytic treatment, as soon as possible within $12 \mathrm{~h} \mathrm{[1]}$, has demonstrated reduced mortality and morbidity rates. Internationally, best practice guidelines recommend reperfusion therapy, but implementation of these guidelines is a critical challenge in resource-poor settings. 
http://wjst.wu.ac.th

Coronary heart disease has been an important cause of morbidity and mortality in Thailand and has been continuously listed as one of the top 5 causes of death. In the 76 provinces of Thailand, in some provincial hospitals in rural areas, approximately 60 - 100 cases per year of STEMI have been reported $[2,3]$. Studies of STEMI patients' access to treatment in Thailand have shown that the time taken by patients from the onset of symptoms to arriving at a hospital ranged from 2 to $20 \mathrm{~h}$, whereas the time from arrival at a hospital to receiving treatment was 29 to $140 \mathrm{~min}$. Therefore, it is important to consider patient, provider, and health system factors inhibiting and enabling access to reperfusion therapy within a situational context.

In metropolitan areas, comprehensive treatments, including thrombolytic therapy, PCI, and advanced medical technology are more accessible and available than in rural areas. Although the use of coronary interventions has increased in most Thai rural hospitals, many do not have the facilities to perform both thrombolytic therapy and PCI [4]. To enhance accessibility of care and reduce the mortality rate for people in rural areas, thrombolytic treatment has been offered in 24 rural hospitals since 2002. These hospitals are identified as thrombolysis-capable hospitals in which general physicians and nurses, and electrocardiogram and cardiac marker laboratories, are allocated as the best available resources for STEMI management with thrombolytic treatment [5].

Studies have shown that access to thrombolytic reperfusion therapy is not optimal, particularly in rural areas [6]. Reports from rural hospitals have shown a variation of mortality from STEMI in the range of 15 to $31.6 \%$, whereas the national mortality from STEMI is $5.3 \%$. Although international practice guidelines recommend that door to needle time should be less than $30 \mathrm{~min}$, only 10 to $30 \%$ of STEMI patients in Thai rural areas reached this goal. Moreover, most studies focus on outcomes of STEMI management in terms of mortality, morbidity, and time of treatment access. There is a lack of studies examining outcomes sensitive to thrombolytic reperfusion.

To improve health outcomes, patients not only have to reach the medical unit, they must also receive appropriate services at the right time. The relationship between health service received, personal characteristics, and healthcare utilization behavior of the patients and patient health outcomes represents a complex and multifaceted interaction [7]. Patients who correctly responded to their cardiac symptoms when having an ACS had a higher survival rate than those who did not [8-10], whereas the witness response to cardiac symptoms of patients can influence time to treatment [11]. Patients with a lower severity of cardiac symptoms at hospital arrival had a lower mortality. Furthermore, decreased prehospital delay and access to definitive treatment contributed to positive patient outcomes [12-14].

Ten years after the introduction of thrombolytic treatment for STEMI patients living in rural areas in Thailand, there is still limited quality outcome data and limited understanding of factors contributing to thrombolytic reperfusion in rural STEMI patients. Therefore, the researchers were interested in studying factors contributing to the clinical outcomes of thrombolytic treatment for STEMI in rural hospitals. The findings could ultimately be used to inform programs to improve the quality of healthcare services.

\section{Materials and methods}

A cross-sectional study was performed to analyze both hospital and patient data in order to understand the factors influencing access to thrombolytic treatment in individuals admitted to medical units at 12 thrombolysis-capable hospitals in rural areas throughout Thailand. The analysis presented in this study focused on rural sites. The eligible settings for this study were selected by a stratified random sampling method. For the first strata, 24 rural hospitals were identified as thrombolysis-capable hospitals by the National Health Security organization, located in four regions of Thailand. Each region has 6 thrombolysis-capable hospitals. For the second strata, 3 from 6 hospitals were randomly selected from each region. Therefore, 12 thrombolysis-capable hospitals were selected. The participants in each selected hospital were purposely selected according to the following inclusion criteria: 1) they were male and female patients with a confirmed diagnosis of STEMI; 2) age > 18 years; 3) they had received thrombolytic drugs; 4) they had agreed to participate, as specified by consent form. The participants in this study were 87 cases, although the calculated sample size was 90 . This followed the recommendation for logistic regression sample size that mandated 15 events per variable as the optimal ratio [15]. During 
the inclusion period, 138 patients were diagnosed as STEMI. Fifty one patients were excluded because they had not received thrombolytic treatment. This non-receipt of thrombolytic treatment was because: duration of onset symptoms to ED was more than $6 \mathrm{hrs}$. $(\mathrm{n}=19)$; they had suffered cardiac arrest on arrival $(n=9)$; they had undergone cardiogenic shock $(n=10)$; their age was more than 80 years $(n=10)$; they refused thrombolytic drugs $(\mathrm{n}=2)$; they had recurrent STEMI within 1 year after previous thrombolytic treatment $(n=1)$. This is indicated in Figure 1.

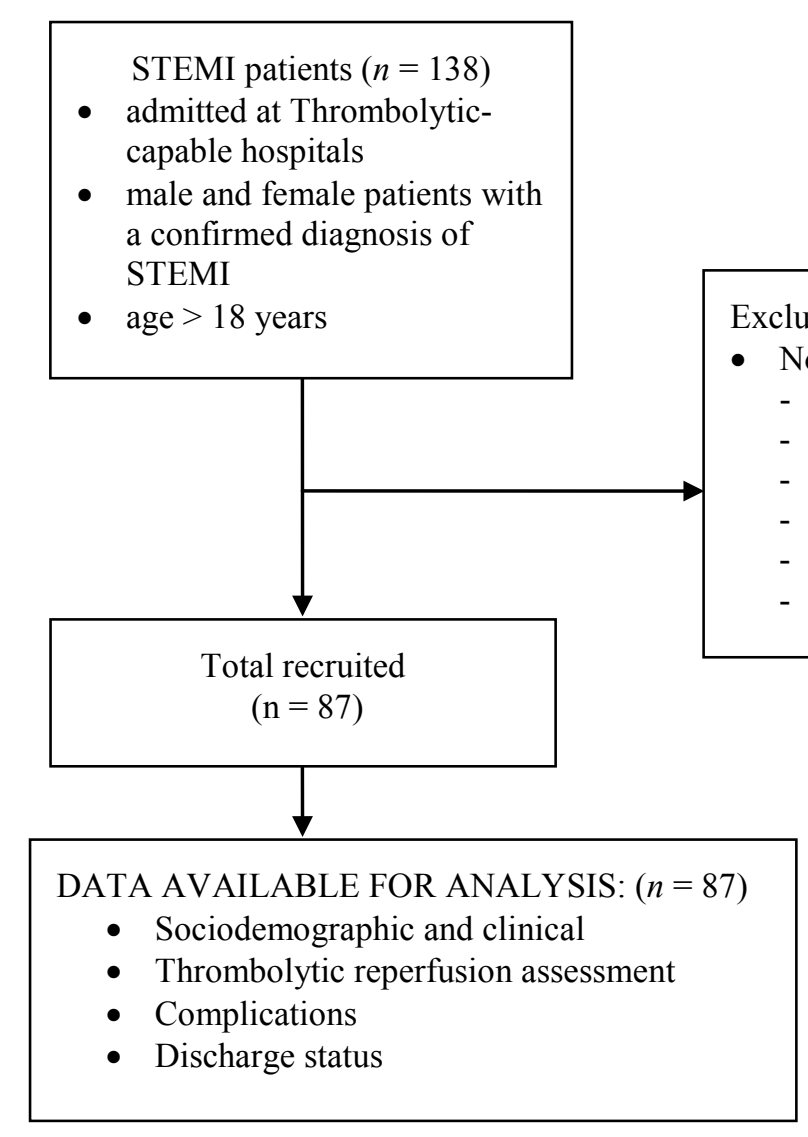

Figure 1 Demonstrate of participants in the cross-sectional study, number of patients enrolled during admission, at discharge. STEMI, ST-Elevation Myocardial Infarction.

Ethics approval was obtained from the Institutional Review Board for research ethics from Mahidol University (MU-IRB / C 2010 / 64.0505 dated June 29 ${ }^{\text {th }}$ 2010). Data collection was started after permission from the research committee and emergency department (ED) of each clinical setting. Emergency nurses coordinated with the researcher when STEMI patients presented at the ED. To avoid any feeling of being forced to participate, the nurse of each hospital asked for the patient's permission to be visited by the researcher. All consenting participants were interviewed when a family member was available to help the participants complete the study instruments. Patients were approached on the seventh day after receiving thrombolytic treatment to retrospectively collect demographic data, clinical characteristics, and treatment outcomes. Following permission the participant was approached by the research team. The researcher introduced herself, described the research objectives and data collection 
http://wjst.wu.ac.th

procedures, and asked for the patient's cooperation. After the patient agreed and signed the consent form to participate in the study, the researcher read the questionnaires or the patient answered independently. Patients had already received thrombolytic treatment before collecting the data; therefore, the process of treatment was not influenced by the research process. No patients refused to participate in the study after the researcher explained about the confidentiality of information and that data would not be individually reported. The quality of the patient records were checked by the patient care team (PCT) of each hospital, and then the researcher extracted the data using standardized definitions.

Thrombolytic reperfusion was the dependent variable and was defined as ST-segment resolution, defined as at least a $50 \%$ decrease in the ST-segment observed within 90 min after receiving thrombolytic therapy. A dichotomous score was used: No (0) for less than $50 \%$ and Yes (1) for at least a $50 \%$ decrease of the ST-segment. To measure the ST-segment resolution, all leads were summed using the ST elevation assessment technique. The ECG interpretations before and after the patient received thrombolytic treatment for 90 min were assessed by an analysis of ST-segment from 12-lead ECG record (using the sum of millimeters or millivolts of ST segment deviation from isoelectric line or baseline of ECG) [16]. All results of ST-segment resolution were analyzed and confirmed with a cardiologist. If any ECG interpretations were inconsistent with the interpretation from a cardiologist, a second opinion from another cardiologist was required. The researcher would clarify the mistake with the cardiologist and start the next data collection procedure.

The study focused on six independent variables as follows: First, patient response and treatment seeking behavior referred to patient behaviors in response to the onset of ACS. Scores were rated by comparing the responding behaviors to the recommended behaviors as set by the 2013 American College of Cardiology Foundation /American Heart Association (ACCF / AHA) guidelines for STEMI [1]. The three behaviors were used for scoring with various weights as follows: 1) 1 point for resting when symptoms occurred; 2) 2 points for taking a sublingual nitroglycerine as soon as possible after symptoms had occurred; and 3) 3 points for calling an ambulance if symptoms had not been relieved within 5 min. The summed score ranged from 0 to 6 . A higher score reflected a higher level of accuracy of ACS response and treatment seeking behavior. Second, witness response to patient symptoms included witness behaviors in response to the patient experiencing onset of ACS. Scores were rated by comparing the responding behaviors to the recommended behaviors as specified in the 2013 ACCF / AHA guidelines for STEMI. The three behaviors were used for scoring and included the following weights: 1) 1 point for recommending the patient go to hospital; 2) 2 points for taking the patient to hospital; and; 3 ) 3 points for calling emergency services to take the patient to the hospital by ambulance immediately. The summed scores ranged from 0 to 6 . A higher score reflected a higher level of appropriateness of ACS response behavior. Third, the severity of illness was evaluated by using the Thrombolysis in Myocardial Infarction (TIMI) Risk score for STEMI [17]. A list of 8 risks was used for scoring and included the following weights: 1) Age was weighted in 2 strata, with 2 points for an age range from 65 to 74 years and 3 points for the age equal to or more than 75 years; 2) 1 point for underlying disease (diabetes, hypertension or angina), and for the results of physical examination; 3) 3 points for blood pressure less than $100 \mathrm{mmHg}$; 4) 2 points for heart rate more than 100 ; 5) 2 points for Killip II-IV; 6) 1 point for body weight less than 67 kilograms; 7) 1 point for ECG present anterior ST-segment elevation or Left Bundle Branch Block (LBBB); and 8) 1 point for onset-to-treatment time more than $4 \mathrm{~h}$. Therefore, the possible range of the summed scores was 0 - 14. A higher score indicated greater severity of illness. Fourth, duration of hospital arrival referred to the time interval from the onset of ACS to hospital arrival measured in minutes. Fifth, waiting time for treatment identified the time interval from hospital arrival to start of thrombolytic treatment measured in minutes as door to needle time. Sixth, received treatment according to the guidelines refers to the thrombolytic reperfusion treatments received by the patients. Scores were rated by comparing the received treatments and the expected treatments recommended by the 2013 ACCF/AHA guidelines for STEMI. The recommended treatments included the following: after arriving at the hospital, 12 lead ECGs and clinical assessment and management should be done in the first 10 min, and then the thrombolytic agent should be started within $30 \mathrm{~min}$. The scores ranged from 0 to 12; a higher score indicated a higher level of accuracy of treatment received. 


\section{Statistical analysis}

Descriptive statistics were used to describe characteristics of the participants in the study. Continuous variables were expressed as means and standard deviations, and categorical variables were expressed as frequencies and percentages. Prior to the study hypothesis testing, the differences of categorical variables were examined using Chi-square and continuous variables were examined using ttest between the thrombolytic reperfusion and non-thrombolytic reperfusion groups. Then, the relationships between severity of illness, patient response and treatment seeking behavior, witness response to the patient symptoms, waiting time for treatment, received treatment according to the guidelines, duration of hospital arrival, and thrombolytic reperfusion signs were examined using logistic regression analysis. Values were considered statistically significant if the P value was $<0.05$. All tests were analyzed using the software program Statistical Program for the Social Sciences (SPSS) version 23.0.

Table 1 Patient demographics and clinical characteristics.

\begin{tabular}{|c|c|c|c|c|}
\hline Variables & $\begin{array}{c}\text { Total sample } \\
n=87(100 \%)\end{array}$ & $\begin{array}{c}\leq 50 \% \text { ST-segment } \\
\text { resolution at } 90 \\
\text { min } \\
n=45\end{array}$ & $\begin{array}{c}>50 \% \mathrm{ST}- \\
\text { segment resolution } \\
\text { at } 90 \mathrm{~min} \\
n=42\end{array}$ & $p$-value \\
\hline Age $(\mathrm{yrs})$ mean $\pm \operatorname{Std}(\min -\max ) \dagger$ & $60.71 \pm 11.99(37-87)$ & $61.73 \pm 11.70(37-87)$ & $59.62 \pm 12.34(40-83)$ & 0.598 \\
\hline \multicolumn{5}{|l|}{ Gender } \\
\hline Female & $27(31.03 \%)$ & 19 & 8 & \\
\hline Male & $60(68.97 \%)$ & 26 & 34 & $0.020 *$ \\
\hline \multicolumn{5}{|l|}{ Underlying diseases ${ }^{a}$} \\
\hline Hypertension & $33(37.93 \%)$ & 20 & 13 & 0.877 \\
\hline Diabetes mellitus & $16(18.39 \%)$ & 14 & 2 & $0.018 *$ \\
\hline Dyslipidemia & $31(35.63 \%)$ & 25 & 6 & $0.002 *$ \\
\hline Coronary artery disease & $36(41.38 \%)$ & 22 & 14 & 0.697 \\
\hline \multicolumn{5}{|l|}{ Transportation method } \\
\hline By own car/taxi & $74(85.06 \%)$ & 36 & 38 & \\
\hline Emergency medical service & $13(14.94 \%)$ & 9 & 4 & 0.244 \\
\hline \multicolumn{5}{|l|}{ Door to needle time } \\
\hline$\leq 30 \min$ & $31(35.63 \%)$ & 17 & 14 & \\
\hline$>30 \mathrm{~min}$ & $56(64.36 \%)$ & 28 & 28 & 0.665 \\
\hline \multicolumn{5}{|l|}{ Complications after SK ${ }^{\mathrm{b}}$} \\
\hline Bleeding & $16(18.39 \%)$ & 7 & 9 & 0.262 \\
\hline Life-threatening arrhythmias & $18(20.69 \%)$ & 12 & 6 & 0.320 \\
\hline Cardiogenic shock & $31(35.63 \%)$ & 23 & 8 & $0.012 *$ \\
\hline Heart failure & $31(35.63 \%)$ & 25 & 6 & $0.001 *$ \\
\hline Stroke & $1(1.15 \%)$ & 1 & 0 & 0.376 \\
\hline Length of hospital stay (day) $\dagger$ & $4.98 \pm 3.04(1-21)$ & $4.71 \pm 3.51(1-21)$ & $5.26 \pm 2.46(1-16)$ & 0.402 \\
\hline \multicolumn{5}{|l|}{ Discharge status } \\
\hline Death & $10(11.49 \%)$ & 9 & 1 & \\
\hline Alive & $69(79.31 \%)$ & 30 & 39 & \\
\hline Refer for rescue PCI & $8(9.20 \%)$ & 6 & 2 & $0.009 *$ \\
\hline
\end{tabular}

Categorical variables are expressed as $n(\%)$, and between-group differences were analyzed using Chi-square tests; Continuous variables are expressed as $\uparrow$ mean \pm standard deviation and between groups difference by t-test.; * highlights significant results; SK, Streptokinase; PCI, Percutaneous Coronary Intervention. $p$-value $<0.05$

${ }^{\mathrm{a}, \mathrm{b}}$ Sum of percentages is not equal to 100 as some subjects had more than one underlying disease and complication. 


\section{Results}

Patient socio-demographic and clinical characteristics

The age of the patients ranged from 37 to 87 years, with an average age of 60.71 years (SD 11.99 years). The majority were male $(68.97 \%)$, were married $(72.41 \%)$, and had a primary-level education $(62.10 \%)$. The majority of the participants $(64.5 \%)$ had an income of less than 100 USD per month. 55.1 $\%$ of the patients were more than 60 years old and $9.4 \%$ were more than 80 years old. This may be because in rural areas elderly people receive basic education and work in agriculture, or some of them cannot work but receive only economic support from their family members, resulting in less income. A total of 87 STEMI patients were enrolled in this study, and $48.28 \%$ of the patients had at least a $50 \%$ decrease in the ST-segment. As shown in Table 1, only $35.63 \%$ of participants received thrombolytic treatment within $30 \mathrm{~min}$ according to the recommendations of the standard guideline. Forty-two patients $(48.28 \%)$ experienced myocardial reperfusion within $90 \mathrm{~min}$ after thrombolytic treatment. Ten patients $(11.49 \%)$ died, whereas 69 patients $(79.31 \%)$ were discharged with improved conditions. In the cases of the dead, the researcher asked for permission to interview and collect data from their relatives who stayed with the patient while symptomatic and who brought them to the hospital. Additionally, patients with underlying diabetes $(18.39 \%)$ and dyslipidemia $(35.63 \%)$ were at higher risk of unsuccessful thrombolytic reperfusion with more cardiogenic shock $(35.63 \%)$ and heart failure $(35.63 \%)$. The characteristics of predicting factors are summarized in Table 2.

Table 2 Characteristics of continuous variable factors during access to treatment $(n=87)$.

\begin{tabular}{|c|c|c|c|c|}
\hline Continuous variable factors & $\begin{array}{l}\text { Total sample } \\
\quad(n=87)\end{array}$ & $\begin{array}{c}\leq 50 \% \\
\text { ST-segment } \\
\text { resolution } \\
\text { at } 90 \text { min } \\
(n=45)\end{array}$ & $\begin{array}{c}>50 \% \\
\text { ST-segment } \\
\text { resolution } \\
\text { at } 90 \text { min } \\
(n=42)\end{array}$ & $p$-value \\
\hline $\begin{array}{l}\text { Patient response and treatment } \\
\text { seeking behaviors }\end{array}$ & $1.90 \pm 1.64(0-6)$ & $1.40 \pm 1.42$ & $2.43 \pm 1.71$ & $0.003 *$ \\
\hline Severity of illness & $4.64 \pm 1.93(0-13)$ & $5.64 \pm 2.85$ & $3.57 \pm 2.63$ & $0.001 *$ \\
\hline Duration of hospital arrival & $215.82 \pm 168.26(9-1247)$ & $228.13 \pm 133.08$ & $202.62 \pm 200.08$ & $0.035^{*}$ \\
\hline Waiting time for treatment & $54.87 \pm 53.92(10-340)$ & $63.71 \pm 69.92$ & $45.40 \pm 25.97$ & 0.591 \\
\hline $\begin{array}{l}\text { Received treatment according to } \\
\text { the guidelines }\end{array}$ & $7.86 \pm 1.61(4-11)$ & $7.27 \pm 1.56$ & $8.50 \pm 1.42$ & $<0.0001 *$ \\
\hline
\end{tabular}

$p$-value $<0.05 ; *$ highlights significant results.

All variables are expressed as mean \pm standard deviation (range) and between-group differences were analyzed using $t$-tests.

\section{Factors predicting thrombolytic reperfusion}

For screening steps, Univariate analysis revealed that seven variables had p-value $<0.15$ which were considered for potential factors: gender, underlying disease DM, dyslipidemia, patient response to patient symptoms, severity of illness, waiting time for treatment, and received treatment according to the guidelines (Table 3), which were then analyzed by multiple logistic regression analysis. However, underlying diseases are the components of severity of illness; therefore, diabetes mellitus and dyslipidemia were excluded from the model. 
http://wjst.wu.ac.th

Table 3 Binary logistic regression analysis of factors predicting thrombolytic reperfusion $(n=87)$.

\begin{tabular}{|c|c|c|c|c|c|}
\hline \multirow[b]{2}{*}{ Variables } & \multicolumn{2}{|c|}{ Univariate } & \multicolumn{3}{|c|}{ Multiple regression Adjusted OR } \\
\hline & $\begin{array}{l}\text { Odds Ratio } \\
\text { (95\% CI) }\end{array}$ & $\begin{array}{c}P- \\
\text { Value }\end{array}$ & $\begin{array}{l}\text { Beta } \\
\text { (SE) }\end{array}$ & $\begin{array}{l}\text { Odds Ratio } \\
(95 \% \text { CI) }\end{array}$ & $P$-Value \\
\hline Age & $0.98(0.95-1.02)$ & 0.410 & & & \\
\hline \multicolumn{6}{|l|}{ Gender } \\
\hline Male & Reference & & & & \\
\hline Female & $0.32(0.12-0.85)$ & $0.022 *$ & $\begin{array}{l}0.41 \\
(0.62)\end{array}$ & $1.51(0.44-5.10)$ & 0.512 \\
\hline \multicolumn{6}{|l|}{ Underlying disease } \\
\hline No hypertension & Reference & & & & \\
\hline Hypertension & $0.85(0.35-2.02)$ & 0.706 & & & \\
\hline No diabetes mellitus & Reference & & & & \\
\hline Diabetes mellitus & $0.14(0.03-0.66)$ & $0.013^{*}$ & \multirow{2}{*}{\multicolumn{3}{|c|}{ Component of Severity of illness }} \\
\hline No dyslipidemia & Reference & & & & \\
\hline Dyslipidemia & $5.56(1.97-15.66)$ & $0.001 *$ & \multicolumn{3}{|c|}{ Component of Severity of illness } \\
\hline No coronary artery disease (ref.) & Reference & & & & \\
\hline Coronary artery disease & $0.73(0.30-1.80)$ & 0.450 & & & \\
\hline \multicolumn{6}{|l|}{ Transformation method } \\
\hline Emergency medical service (ref.) & Reference & & & & \\
\hline By own car/taxi & $1.91(0.54-6.77)$ & 0.314 & & & \\
\hline \multicolumn{6}{|l|}{ Patient response and treatment } \\
\hline Witness response to patient symptoms & $1.23(0.85-1.76)$ & 0.270 & & & \\
\hline Severity of illness & $0.75(0.63-0.90)$ & $0.002 *$ & $-0.26(0.10)$ & $0.77(0.64-0.94)$ & $0.009^{* *}$ \\
\hline $\begin{array}{l}\text { Received treatment according to the } \\
\text { guidelines }\end{array}$ & $1.74(1.26-2.41)$ & $0.001 *$ & $0.46(0.18)$ & $1.58(1.12-2.25)$ & $0.010 * *$ \\
\hline Duration of hospital arrival & $0.10(0.99-1.00)$ & 0.484 & & & \\
\hline Waiting time for treatment & $0.99(0.98-1.00)$ & $0.147^{*}$ & $0.00(0.00)$ & $1.00(0.99-1.01)$ & 0.861 \\
\hline
\end{tabular}

$p$-value $<0.15 ; *$ highlights significant results of Univariate analysis.

$p$-value $<0.05 ; * *$ highlights significant results of multiple regression analysis.

Note: -2 Log likelihood $=80.26$, Nagelkerke $\mathrm{R}^{2}=0.36$

Multivariate analysis revealed that patient response and treatment seeking behavior, witness response to patient symptoms, severity of illness, duration of hospital arrival, waiting time for treatment, and having received treatment according to the guidelines were continuous variable factors predicting thrombolytic reperfusion. However, not all of the variables in this study were statistically significant at the 0.05 level, as shown in Table 3.

The main finding of the study was that there were significant relationships between thrombolytic reperfusion and patient response and treatment seeking behavior (Odds ratio 1.47, 1.01 - 2.04), severity of illness (Odds ratio 0.77, 0.64 - 0.94), and having received treatment according to the guidelines (Odds ratio $1.58,1.12-2.25)$. For each score of patient response and treatment seeking behavior, the odds of myocardial reperfusion increased by 1.47 . This finding is consistent with the finding of comparative testing between the successful and low myocardial reperfusion groups. The mean score of patient response to symptoms in the low myocardial reperfusion group (1.40 \pm 1.42$)$ was significantly less than that of the successful group (2.43 \pm 1.71$)$.

The mean waiting time for thrombolytic treatment was longer than the recommended time $(54.87 \pm 53.92)$. However, the waiting time for treatment in the successful myocardial reperfusion group (45.40 \pm 25.97$)$ was less than that for the poor reperfusion group (63.71 \pm 69.92$)$. Severity of illness significantly predicted poor myocardial reperfusion. For every 1 score increment in severity of illness, the odds of thrombolytic reperfusion decreases $23 \%$. The patients with poor myocardial reperfusion had a higher score of severity of illness $(5.64 \pm 2.85)$ than the successful reperfusion group $(3.57 \pm 2.63)$, as shown in Table 2. Guideline adherence had a significantly positive effect on thrombolytic reperfusion. 
http://wjst.wu.ac.th

The patients who received thrombolytic treatment within $30 \mathrm{~min}$ after arrival at the ED were found to achieve thrombolytic reperfusion 1.58 times greater than those who did not (95\% CI $1.12-2.25)$, as shown in Table 3.

\section{Discussion}

This is the first study in Thailand examining the association between patient characteristics and hospital factors influencing thrombolytic reperfusion in patients with STEMI after receiving thrombolytic treatment in a usual care setting in rural hospitals.

In this study, the proportion of STEMI patients who received thrombolytic treatment within $30 \mathrm{~min}$ according to the recommendations of the standard guidelines is higher than that reported by other investigators. The range of door to needle time within $30 \mathrm{~min}$ was 10 to $22.2 \%$ among thrombolysiscapable hospitals [3,6]. There has been an increase in treatment access, but there is still a great need for improvement. The mortality of patients receiving treatment in thrombolysis-capable hospitals in this study was less than previous studies, which had a range from 15.0 to $31.6 \%$. Approximately $5.3 \%$ died from STEMI in metropolitan and large cities [2]. Most of the deaths were in the poor thrombolytic reperfusion group. This observation was comparable to previous studies, which reported that general characteristics of STEMI patients who were at risk of high mortality included being older than 60 years, being of female gender, and having underlying coronary artery disease and diabetes [18].

Patients with higher coronary reperfusion had less comorbidity, which is consistent with previous literature indicating that STEMI patients having underlying diseases contributed to greater severity of illness. Previous studies have found that patients with a history of chest pain, unstable angina, diabetes mellitus, hypertension, and heart failure often delayed seeking health care and had high mortality rates after treatment $[19,20]$. This result challenges health service providers to identify strategies to increase the adherence of these high-risk patients [21]. In this study, the individuals living in a rural area with chronic conditions did not regularly follow their treatment due to lack of awareness of the disease and treatment, the use of traditional therapies, financial concerns, and misunderstanding of their healthcare.

The statistically significant relationships between thrombolytic reperfusion and patient response, treatment seeking behavior, severity of illness, and receiving treatment according to the guidelines are supported by previous research data indicating that there have been significantly better outcomes where there was rapid access to thrombolytic treatment among patients with STEMI [22]. However, in this study, gender, witness response to patient symptoms, time taken for hospital arrival, and waiting times for treatment were not significant predictors of thrombolytic reperfusion.

This finding is consistent with the ACC/AHA guideline recommending early arrival to the hospital if the symptoms are not relieved by rest and nitroglycerine. The most frequent behavior of patient response to symptoms in this study was to stop their activities and tell someone to take them to the hospital by their own car and not call for an ambulance. Each decreased score of patient response and treatment seeking behavior reduced the chance of successful myocardial reperfusion; in this study, participants did not stop their activity when the symptoms occurred but rather used strategies to decrease symptoms, such as massage, drinking coffee, and ingesting antacid drugs. However, previous studies suggest that patients who appropriately respond to the symptoms and arrive at the hospital earlier will have improved survival rates $[23,24]$. In this study, the use of emergency medical services (EMS) was only $14.9 \%$. This is an important challenge for healthcare providers to identify effective strategies for high-risk patients and the public to improve treatment seeking behaviors, especially calling for an ambulance to decrease transportation time and receive effective pre-hospital management from a dispatcher center [25].

The recommendations for actions of witnessing bystanders should include suggesting the patient go to the hospital, taking the patient to the hospital without delay, or calling an ambulance immediately. However, in this study, most of the witnesses took the patient to hospital in their own car. Some of them tried to relieve symptoms and find suggestions for treatment from other people. Therefore, these results are consistent with previous studies which point out the need for the health service system to increase public awareness of this issue. 
http://wjst.wu.ac.th

In this study, some participants delayed more than $4 \mathrm{~h}$ before arriving at a thrombolytic-capable hospital. They firstly presented at the community hospital, primary care units, or private clinics, before being referred to a thrombolysis-capable hospital. This increased transportation time before accessing definitive treatment. This finding challenges health service to consider effective strategies to increase ACS awareness and ensure appropriate responses by patients and families so that the patient has timely access to definite health service when ACS symptoms attack. Healthcare providers, especially nurses who work with high risk patients such as the elderly, women, or patients with chronic diseases such as CAD, HT, dyslipidemia and DM, must deliver clear information for decision-making and prepare an individual action plan for each patient and their family to ensure timely access health service in the emergency period. These strategies are particularly important for individuals located in remote areas.

Severity of illness significantly predicted poor myocardial reperfusion. Severity of illness in this study was measured by TIMI risk score for STEMI, which weighted the risk score by age over 65 years; this group accounted for more than 40 percent of the study sample. Moreover, all participants showed ST elevation on their electrocardiograms. This study found that the patients with poor myocardial reperfusion were older and had more signs of heart failure and underlying diseases such as diabetes mellitus, and dyslipidemia. Therefore, these patients should be a primary focus for maximizing treatment outcomes and preventing potential complications, such as cardiogenic shock and heart failure.

In previous studies, guideline adherence was found to have a positive effect on thrombolytic reperfusion, with strong evidence that decreasing door to needle time may result in increased thrombolytic reperfusion [26,27]. Many studies have confirmed that a longer duration of waiting time for treatment in the emergency department, especially during crowded situations, was significantly associated with poor outcomes. However, this study found no significant association between waiting time for treatment and thrombolytic reperfusion. This finding could be explained by the fact that every thrombolysis-capable hospital in the study had developed a STEMI fast track policy. Our finding is consistent with the literature, indicating that, even if an emergency department is crowded, it has little impact on patients identified as critical once the ED has well-developed care protocols or clinical practice guidelines [28]. Thus, the challenge for management systems for STEMI patients is to design strategies that will promote and ensure the strict adherence of all hospitals to the standard clinical practice guidelines.

\section{Limitations}

This study was conducted with a small sample size and did not include data from those did not have access to care, or those did not seek healthcare for acute chest pain. These groups are actually the groups who need this healthcare the most. Furthermore, the clinical measurements should have included echocardiographic parameters, but the records were not available in all patients due to the lack of cardiologists and echocardiogram services in rural hospitals. Moreover, pre-hospital management was not included in the study because most patients used self-transportation to the hospital. In spite of these limitations, this study has cast an important spotlight on the importance of care improvement in rural areas of Thailand and other low resource settings.

\section{Conclusions and recommendations}

Although evidence-based guidelines are widely available, contextual issues need to be considered, particularly in rural areas where healthcare resources and transfer facilities are limited. Patient, provider, and health system factors mediate outcomes in STEMI and underscore the need for strengthening health systems. Three significant factors, patient response and treatment seeking behavior, received treatment according to the guidelines, and severity of illness, are important to explain thrombolysis reperfusion. Raising awareness and appropriate symptom management in high-risk groups will decrease delay in accessing health services. Improved management at the emergency room for acute chest pain patients will improve door to needle time. The number of thrombolysis-capable hospitals needs to be increased. In addition, clinical practice guidelines need to be promoted, and pre-hospital factors should be considered 
to increase opportunities for myocardial reperfusion. These factors are highly dependent on a competent and qualified nursing workforce.

\section{Recommendations}

The research findings also highlight the importance of allocating health care resources. Results from the study necessitate further research and practice to increase access to reperfusion treatment in Thailand, as follows. For clinical practice, this includes increasing capacity in thrombolysis-capable hospitals and promoting guideline adherence and the monitoring of clinical parameters within a clinical governance framework. Promoting access to emergency response services will likely improve access to definitive care for STEMI patients in rural areas. In addition, early diagnosis by 12-lead ECG, together with proper management including oxygen therapy, aspirin, and nitroglycerine administration during patient transportation, should be addressed. This represents an important opportunity for advanced practice nurses. Management and education strategies to increase the patient's awareness of receiving thrombolytic treatment are essential. The recognition of these factors by the multidisciplinary healthcare team may be useful in developing an effective health service system for patients with acute myocardial infarction in rural areas. For further study, a replication study with a larger sample size and analysis with multilevel regression analysis, both at health service-level and patient-level, is needed to explain all levels of influence on myocardial reperfusion. Studies promoting strategies to enhance symptoms awareness and appropriate management when acute coronary symptoms occur need to be undertaken on patients and their family members. This is especially important among those patients with hypertension, diabetes, and dyslipidemia, and it is also important to consider the Thai context in such studies.

\section{Acknowledgements}

We would like to sincerely thank all the STEMI patients for their data. Moreover, we thank the Thai Nursing and Midwifery Council for the funding support for this study.

\section{References}

[1] PT O'Gara, FG Kushner, DD Ascheim, DE Jr Casey, MK Chung, JA de Lemos, SM Ettinger, JC Fang, FM Fesmire, BA Franklin, CB Granger, HM Krumholz, JA Linderbaum, DA Morrow, LK Newby, JP Ornato, N Ou, MJ Radford, JE Tamis-Holland, CL Tommaso, CM Tracy, YJ Woo, DX Zhao, JL Anderson, AK Jacobs, JL Halperin, NM Albert, RG Brindis, MA Creager, D DeMets, RA Guyton, JS Hochman, RJ Kovacs, FG Kushner, EM Ohman, WG Stevenson and CW Yancy. 2013 ACCF/AHA guideline for the management of ST-elevation myocardial infarction: A report of the American College of Cardiology Foundation/American Heart Association Task Force on Practice Guidelines. Circulation 2013; 127, 362-425.

[2] S Srimahachota, S Boonyaratavej, R Kanjanavanit, P Sritara, R Krittayaphong, R Kunjara-Naayudhya and P Tatsanavivat. Thai Registry in Acute Coronary Syndrome (TRACS) - An Extension of Thai Acute Coronary Syndrome Registry (TACS) group: Lower in-hospital but still high mortality at one-year. J. Med. Assoc. Thai 2012; 95, 508-18.

[3] P Juntaranimi, S Kiatchoosakun and S Duangsorn. Clinical outcomes of acute ST elevation myocardial infarction patients in a comprehensive cardiac referral system in Khon Kaen province. Thai Heart J. 2011; 24, 62-9.

[4] M Maraprasertsak. Three years experience comparing the fast track system and patient education on ST-segment elevation myocardial infarction in Phrae Hospital. Thai Heart J. 2008; 28, 52-60.

[5] P Lab-ying. 2008. The final report of evaluation project of The developing Cardiac Excellence Center system under National Health Security Scheme. (in Thai). NHSO, HSRI and HISO. Bangkok, Thailand.

[6] P Promlikitchai, P Suchatsuntorn, S Kobkuechaiyapong, T Doungngern and P Doungakka. Using digital ECG consultation system to facilitate cases for ST-elevation MI in Saraburi Hospital. J. Med. Assoc. Thai 2011; 94, 933-40. 
http://wjst.wu.ac.th

[7] RM Anderson. Revisiting the behavioral model and access to medical care: does it matter? J. Health Soc. Behav. 1995; 36, 1-10.

[8] H Brokalaki, K Giakoumidakis, NV Fotos, P Galanis, E Patelarou, E Siamaga and IS Elefsiniotis. Factors associated with delayed hospital arrival among patients with acute myocardial infarction: A cross-sectional study in Greece. Int. Nurs. Rev. 2011; 58, 470-6.

[9] C Henriksson, M Larsson, J Arnetz, M Berglin-Jarlöv, J Herlitz, JE Karlsson, L Svensson, M Thuresson, C Zedigh, L Wernroth and B Lindahl. Knowledge and attitudes toward seeking medical care for AMI-symptoms. Int. J. Cardiol. 2011; 147, 224-7.

[10] MA Abed, RM Ali, MM Abu Ras, FO Hamdallah, AA Khalil and DK Moser. Symptoms of acute myocardial infarction: A correlational study of the discrepancy between patients' expectations and experiences. Int. J. Nurs. Stud. 2015; 52, 1591-9.

[11] L Lesneski. Factors influencing treatment delay for patients with acute myocardial infarction. Appl. Nurs. Res. 2010; 23, 185-90.

[12] R Taha, M Oraby, G Nasr and A El-Hawary. Prevalence and causes of failure of receiving thrombolytic therapy in patients with acute ST-segment elevation myocardial infarction. Egypt. Heart J. 2013; 65, 51-5.

[13] PH Nielsen, CJ Terkelsen, TT Nielsen, L Thuesen, LR Krusell, P Thayssen, H Kelbaek, U Abildgaard, AB Villadsen, HR Andersen and M Maeng. System delay and timing of intervention in acute myocardial infarction (from the Danish Acute Myocardial Infarction-2 [DANAMI-2] trial). Am. J Cardiol. 2011; 108, 776-81.

[14] BR Nascimento, MR de Sousa, FN Demarqui and AL Ribeiro. Risks and benefits of thrombolytic, antiplatelet, and anticoagulant therapies for ST segment elevation myocardial infarction: Systematic review. ISRN Cardiology. 2014; 2014, 416253.

[15] E Núñez, EW Steyerberg and J Núñez. Regression modeling strategies. Rev. Esp. Cardiol. 2011; 64, $501-7$.

[16] JS Crowder, MW Hubble, S Gandhi, H McGinnis, S Zelman, W Bozeman and J Winslow. Prehospital administration of tenecteplase for ST-segment elevation myocardial infarction in a rural EMS system. Prehosp. Emerg. Care 2011; 15, 499-505.

[17] DA Morrow, EM Antman, A Charlesworth, R Cairns, SA Murphy, JA de Lemos , RP Giugliano, $\mathrm{CH}$ McCabe and E Braunwald. TIMI risk score for ST-elevation myocardial infarction: A convenient, bedside, clinical score for risk assessment at presentation. Circulation 2000; 102, 2031-7.

[18] E Kalinauskiene, D Gerviene, I Sabeckyte and A Naudziunas. Characteristics and outcomes of patients with acute myocardial infarction at non-PCI Capable Hospitals in 2007 and in 2014. Bio. Med. Res. Int. 2015; 2015, 359372.

[19] PM Davidson, J Daly, D Leung, E Ang, G Paull, M DiGiacomo, K Hancock, Y Cao, H Du and DR Thompson. Health-seeking beliefs of cardiovascular patients: a qualitative study. Int. J. Nurs. Stud. $2011 ; 48,1367-75$.

[20] JJ Khan, JW Albarran, V Lopez and SY Chair. Gender differences on chest pain perception associated with acute myocardial infarction in Chinese patients: a questionnaire survey. J .Clin. Nurs. 2010; 19, 2720-9.

[21] S Sindhu, C Pholpet and S Puttapitukpol. Meeting the challenges of chronic illness: A nurse-led collaborative community care program in Thailand. Collegian 2010; 17, 93-9.

[22] A Kobayashi, N Misumida, S Aoi, E Steinberg, K Kearney, JT Fox and Y Kanei. STEMI notification by EMS predicts shorter door-to-balloon time and smaller infarct size. Am. J. Emerg. Med. 2016; 34, 1610-3.

[23] P Thepphawan, D Watthnakitkrileart, K Pongthavornkamol and C Dumavibhat. Cognitive representation, emotional responses and hospitalization experience in predicting decision making for receiving treatment among patients with acute coronary syndrome. J. Nurs. Sci. 2011; 29, 111-9.

[24] H Brokalaki, K Giakoumidakis, NV Fotos, P Galanis, E Patelarou, E Siamaga and IS Elefsiniotis. Factors associated with delayed hospital arrival among patients with acute myocardial infarction: A cross-sectional study in Greece. Int. Nurs. Rev. 2011; 58, 470-6. 
http://wjst.wu.ac.th

[25] LD Kinsman, T Rotter, J Willis, PC Snow, P Buykx and JS Humphreys. Do clinical pathways enhance access to evidence-based acute myocardial infarction treatment in rural emergency departments? Aust. J. Rural Health 2012; 20, 59-66.

[26] DJ Hsieh and WK Chen. Quality of care of patients presenting with acute coronary syndrome in emergency departments in Taiwan. J. Acute Med. 2011; 1, 33-40.

[27] AA Motivala, P Christopher, P Cannon, VS Srinivas, D Dai, AF Hernandez, ED Peterson, DL Bhatt and GC Fonarow. Changes in myocardial infarction guideline adherence as a function of patient risk: An end to paradoxical care? J. Am. Col. Cardiol. 2011; 58, 1760-5.

[28] B Harris, J Bai and EB Kulstad. Crowding does not adversely affect time to percutaneous coronary intervention for acute myocardial infarction in a community emergency department. Ann. Emerg. Med. 2012; 59, 13-7. 